\title{
Throttling Aperture as the Gaseous Secondary Electron Detector in the Variable Pressure/Environmental SEM
}

\author{
M. KRYSZTOF* AND W. SŁóWKO \\ Faculty of Microsystem Electronics and Photonics, Wrocław University of Technology \\ Z. Janiszewskiego 11/17, 50-372 Wrocław, Poland
}

\begin{abstract}
The authors analysed the conditions of the secondary electron detection with the use of the lower throttling aperture as the electron collector positioned close to the sample, at the distance less, or comparable to the aperture opening. For computer simulations of electron flow under various gas conditions, the software combining the commercial programme SIMION 3D v. 7.0, enabling computations of charged particles trajectories in the electric and magnetic fields, combined with the Monte Carlo one written by the authors was used. The results of the simulations show that the gas amplification of the electron signal and the noise to signal ratio are suitable for imaging at working gas pressures exceeding $1 \mathrm{hPa}(100 \mathrm{~Pa})$. The upper limit of the pressure defined by the electron beam scattering by far exceeds $10 \mathrm{hPa}$ and is a question of the accelerating voltage and vacuum units design. The detector unit combined also with the differential pumping system has been designed in the form of an attachment to the classic scanning electron microscopy and applied in the JSM840 (JEOL) microscope. The simulation results have been proved experimentally.
\end{abstract}

DOI: 10.12693/APhysPolA.123.880

PACS: 41.85.-p, 52.80.Dy

\section{Introduction}

The technique of scanning electron microscopy (SEM) allowing gas presence in the sample chamber has already become very appreciated in many areas where the dielectric samples or those containing constituents of high vapour pressures are investigated. The technique was originally called the environmental SEM but since it becomes a trade mark, the name variable pressure SEM or mixed description of variable pressure/environmental SEM (VP/E SEM) has been in common use. Successful development of the technique was a result of numerous inventions and research of Danilatos [1] as well as achievements of many other scientists involved in the refining process. Among many important physical mechanisms applied in VP/E SEM, these connected with the electron signal detection seem particularly important. The signal promising best resolution is created by the secondary electrons (SE) and the so-called gaseous detection device or gaseous secondary electron detector (GSED) $[2,3]$ has been a dominant technique since the beginning. This consists in collecting electron stream multiplied in ionising collisions with gas molecules on the path from the sample to an annular anode placed at the face of the objective lens. As the electron current multiplication in the Townsend discharge strongly depends on the sampleanode distance $d$ and gas pressure $p$ or rather on their product $p d$, the GSED technique fails when this value is too low.

Taking this into consideration, the authors have developed techniques through the lower throttling aperture

*corresponding author; e-mail: michal.krysztof@pwr.wroc.pl
(LTA) secondary electron detection which relies on transportation of the SE signal into the intermediate chamber where it is finally detected with the use of the scintillating technique [4]. To make this process more efficient, the SE stream was focused on the aperture opening by means of an immersion lens formed with the use of the additional aperture called the screening aperture. The concept of the SE stream focusing may be also useful to improve the classic GSED technique in the case of low $p d$ values. The SE detector of this kind has been applied in combination with the directional backscattered electron (BSE) detector destined for three-dimensional imaging of the sample topography in VP/E SEM with the use of the multi-detector method. In this solution, location of the quadrant semiconductor BSE detector in the intermediate chamber imposed the necessity to keep a very low distance between the sample and LTA, even less than the LTA diameter (about $0.6 \mathrm{~mm}$ ). This implies very small $p d$ factor and hard conditions for detection of the SE signal.

\section{Numerical modeling of the electron flow}

To estimate an efficiency of the described mechanism, the authors conducted a series of computer simulations of the electron flow under various conditions with the use of the MC SIMION programme. This software combines the commercially available programme SIMION 3D v.7.0, destined for computations of charged particles trajectories in the electric and magnetic fields, and the Monte Carlo one written by the authors in the SIMION internal language [5]. The MC programme takes into consideration the electron scattering in elastic and inelastic collisions but also the ion flow and secondary electron emission stimulated by ion bombardment may be considered for an inhomogeneous distribution of the gas pressure as 
well. The crucial input data defining the events that occur on the unit path and at the unit gas pressure are introduced to the programme in the form of the electron cross-sections for various kinds of collisions as the functions of electron energies in the assumed working gas. At each calculation step (much shorter than the mean free electron path) probabilities of particular events are calculated on these databases and successive events are sampled.

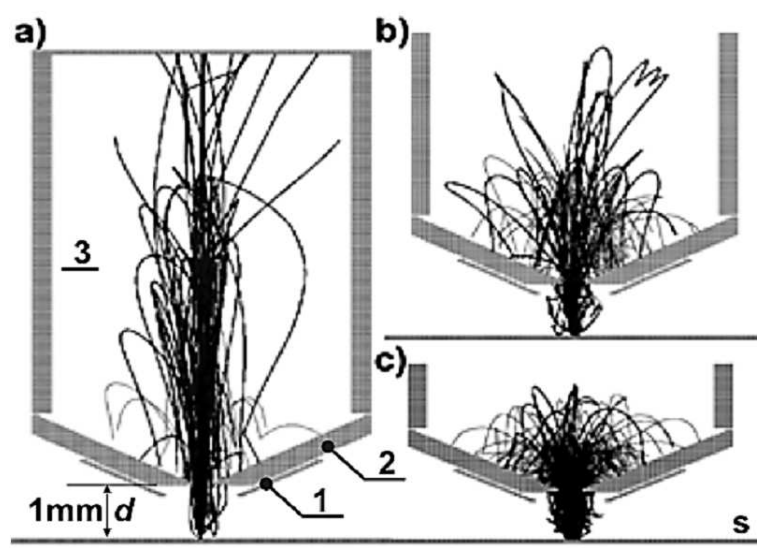

Fig. 1. Trajectories of the secondary electrons simulated at different gas (water vapour) pressures in the sample chamber $(\mathrm{s}$ - sample, 1 - screening aperture, 2 - throttling aperture, 3 - intermediate chamber, $d=1 \mathrm{~mm}-$ gaseous working distance, $U_{\mathrm{TA}}=300 \mathrm{~V}$, $W_{0}=2 \mathrm{eV}$ ): (a) $P_{1}=0.5 \mathrm{hPa}$, (b) $P_{1}=3 \mathrm{hPa}$, (c) $P_{1}=7 \mathrm{hPa}$.

The calculations have been performed in water vapour environment for the simple four electrode model comprising the sample stage (s), the screening aperture (1), the throttling aperture (2) and the intermediate chamber (3) as it is shown in Fig. 1. The first and the next electrodes were grounded while the LTA bias of $U_{\mathrm{TA}}=300 \mathrm{~V}$ and the initial SE energy of $W_{0}=2 \mathrm{eV}$ were assumed. The trajectories calculated for the low working pressure of $0.5 \mathrm{hPa}$ (Fig. 1a) show that a well-focused SE stream accelerated by the LTA bias can penetrate deeply into the intermediate chamber where it is gradually retarded and directed back toward the sample stage and once more back to the LTA until they finally hit the LTA, which essentially elongates the real path covered by electrons and should increase the number of environmental electrons produced by them. At higher pressures both electron scattering and generation of environmental electrons became more and more intensive but thanks to low $d$ (the gaseous working distance) and good focusing most of the multiplied electron stream is collected on the inside surface of the LTA.

\section{Discussion of the calculation results}

To describe the mentioned mechanism of gas multiplication in a quantitative form, the authors performed calculations of electron flow to particular sectors of the LTA. Their results are shown in Figs. 2 and 3 in the form of the multiplication shares defined as numbers of electrons reaching particular LTA sectors related to the initial number of starting electrons for gas pressures in the sample chamber, $P_{1}$ varying from $0.5 \mathrm{hPa}$ to $7 \mathrm{hPa}$ and for three values of the extracting voltage $\left(U_{\mathrm{TA}}=200\right.$, 300 , and $400 \mathrm{~V})$. As follows from Fig. 1 the signal amplification at a short gaseous working distance $d$ may be low, particularly for low gas pressures in the sample chamber but it seems possible to make up for this shortage in large space of the intermediate chamber if the intermediate gas pressure $P_{2}$ is sufficient.
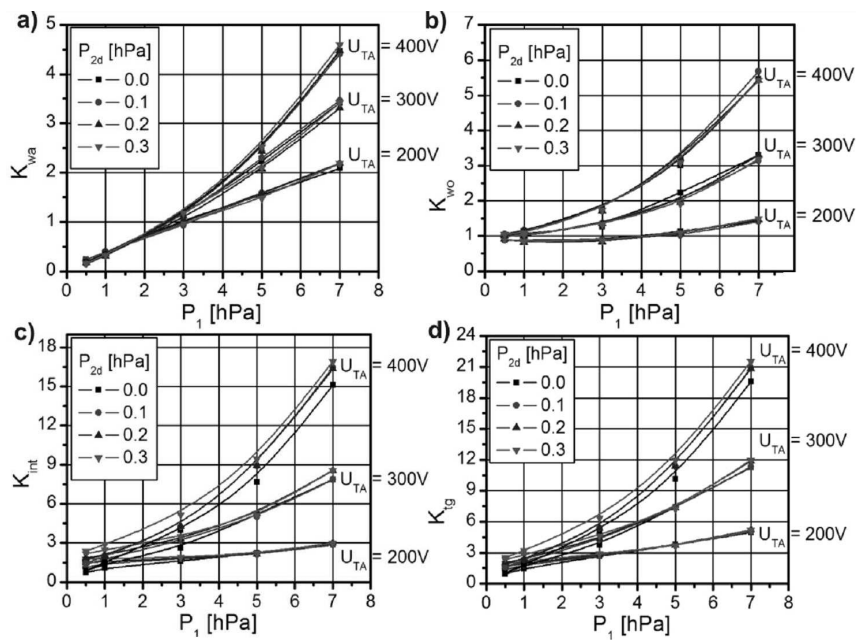

Fig. 2. Amplification shares of the SE signal in particular sectors of the detector at different pressures of the gas dosed into the intermediate chamber $\left(P_{2 d}=0,0.1\right.$, $0.2,0.3 \mathrm{hPa}$ ) and three values of extracting voltages as a function of the working pressure $P_{1}$ : (a) at the face of the throttling aperture, (b) in the aperture opening, (c) at the internal side of the throttling aperture, (d) final $\left(K_{\mathrm{tg}}=K_{\mathrm{wa}}+K_{\text {int }}\right)$.

The gas flow from the sample chamber causes certain pressure distribution inside the intermediate chamber. During the computer calculations the authors took this into consideration dividing the intermediate space into three successively increasing sectors with the pressures $P_{2}=0.4 P_{1}, 0.16 P_{1}$, and $0.1 P_{1}$. These shares may be too low for an effective signal amplification in the range of low gas pressures $P_{1}$ in the sample chamber so the authors tried to dose the additional gas at the pressures $P_{2 d}$ of $0.1,0.2$, and $0.3 \mathrm{hPa}$ into the intermediate chamber, which is also shown in the discussed figures. In Fig. $2 \mathrm{a}$ the signal multiplication share $K_{\text {wa }}$ accomplished by the electrons flowing directly to LTA attains the value of 0.2 at $P_{1}=0.5 \mathrm{hPa}$ which points out to a scarce ionisation avalanche and good focusing which directs most electrons to the LTA opening. The latter fraction marked $K_{\text {wo }}$ is supplementary to the former one and attains the value close to 1 in this point. At the maximum pressure of $7 \mathrm{hPa}$ the mentioned multiplication fractions come to 4.5 


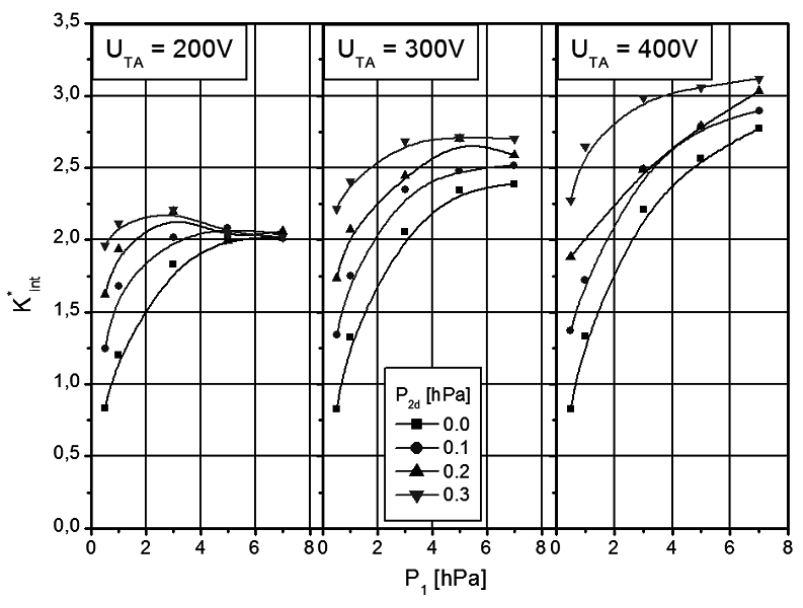

Fig. 3. Amplification of the SE signal inside the intermediate chamber at different pressures of the gas dosed to it $\left(P_{2 d}=0,0.1,0.2,0.3 \mathrm{hPa}\right)$ for three values of extracting voltage $\left(U_{\mathrm{TA}}: 200,300,400 \mathrm{~V}\right)$, as a function of the working pressure in the sample chamber $P_{1}$.

and 5.5 respectively (at $U_{\mathrm{TA}}=400 \mathrm{~V}$ ) which would give the summary signal multiplication of 10 at the distance $d$. The additional gas dosing did not influence these results because its back flow to the sample chamber was not assumed.

The prevailing share of electrons passes LTA and enters the intermediate chamber to be multiplied. There, the internal multiplication coefficient $K_{\text {int }}^{*}$ is defined as a relation of the number of electrons reaching LTA inside the chamber to the number of those incoming the chamber.

The diagrams of $K_{\text {int }}^{*}$ vs. the working gas pressure $P_{1}$ have been shown in Fig. 3 separately for each extracting voltage $U_{\mathrm{TA}}$ because they were crossing over when shown in a common field. The internal multiplication $K_{\text {int }}^{*}$ depends distinctly on the dosed gas pressure $P_{2 d}$ in the range of low sample chamber pressures and depends even more on the extracting voltage $U_{\mathrm{TA}}$. As a result, the multiplication for the maximum values of these parameters $\left(P_{2 d}=0.3 \mathrm{hPa}\right.$ and $\left.U_{\mathrm{TA}}=400 \mathrm{~V}\right)$ changes slightly from 2.3 to 3.2 when $P_{1}$ alters from $0.5 \mathrm{hPa}$ to $7 \mathrm{hPa}$. However, input multiplications depend strongly on the working pressure $P_{1}$ so the final signal multiplication comprising these sector flows also increases rapidly with the $P_{1}$ growth to achieve the maximum multiplication level of 22 times.

It would be possible to obtain higher multiplications particularly at low $P_{1}$ values by dosing more gas into the intermediate chamber. However, excessive gas dosing to the intermediate chamber seems quite impractical for the increasing scattering of both the primary electron beam and the BSE signal detected in this space and an excessive gas leak toward the electron optical column.

On the other hand, GSEDs are not destined for high vacuum applications and they are used most frequently for non-conductive specimens at the working pressure $P_{1}$ about $2 \mathrm{hPa}$. At this pressure gas ionisation is intensive enough to compensate charging effects and the SE signal multiplication (between 3 and 5 here) may be satisfying for good imaging if noise level is kept low.

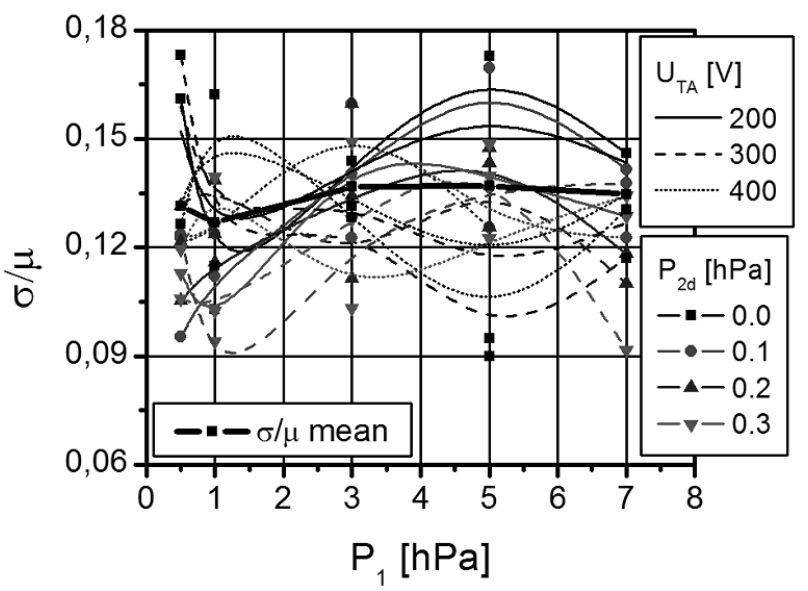

Fig. 4. Standard deviation $\sigma$ to the mean number of electrons $\mu$ ratio as a function of the working pressure in the sample chamber $P_{1}$ on the bases of calculations conducted for the diagrams in Fig. 2d. Thick solid line - the mean level.

The authors assumed generally that the numerical model reflects the reality, which implies also the opinion that statistical fluctuations of the calculations reflect noise accompanying real processes. Thus, the calculations were made for groups of $N_{\mathrm{SE}}=50$ starting electrons and repeated at least 20 times for every point (trial) which enabled to define the mean number of electrons $\mu$ and the standard deviation $\sigma$ for a particular group of electrons. Thus, the data shown in Fig. 4 may be interpreted as the diagrams of noise to the signal ratio, $\mathrm{N} / \mathrm{S}$ (putting $\sigma / \mu \approx \mathrm{N} / \mathrm{S}$ ) vs. the gas pressure (water vapour) at the electron initial energy $W_{0}=2 \mathrm{eV}$. The diagrams were based on the same calculation results as the amplification diagrams displayed in Fig. 2 so they concern the same gas pressures and extracting voltages. Dispersion of particular calculation outcomes is noticeable and probably caused by a relatively low number of trials. The $\sigma / \mu$ values are between 0.07 and 0.17 but their mean level varies slightly from 0.12 to 0.13 (solid thick line) in the whole range of pressures which would be satisfying for good imaging if other noise sources were also low.

\section{Experimental results}

As it has been mentioned at the beginning, the gaseous SE detector discussed above has been applied in combination with a directional four-quadrant BSE detector and the features of the latter one decided about the application scope. The detector unit combined also with the differential pumping system has been designed in the form of an attachment for the classic SEM and applied in the JSM840 (JEOL) microscope. 
Very low distance $d$ to the sample excludes wet biological specimens which might be sucked into the detector working as a vacuum cleaner. So non-conducting solid objects which need gas atmosphere for neutralisation of charging effects are most suitable samples here. The authors conducted a series of experiments concerning imaging conditions suitable for such kind of samples.
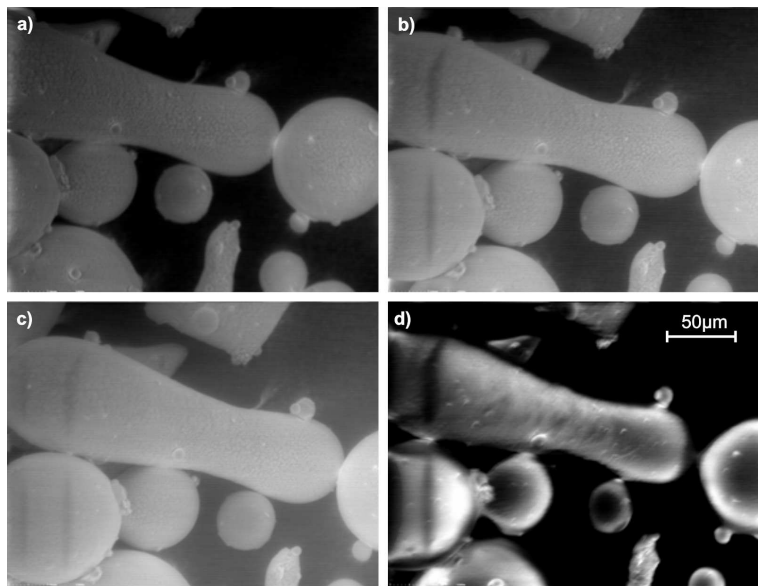

Fig. 5. Ceramic grains on an adhesive tape taken with use of the GSE detector at various gaseous working distances $d$ and gas pressures $P_{1}$ (air, $U_{\mathrm{a}}=15 \mathrm{kV}, \mathrm{WD}=$ $20 \mathrm{~mm}$, horizontal field width $250 \mu \mathrm{m}$ ): (a) $d=0.5 \mathrm{~mm}$, $P_{1}=4 \mathrm{hPa}$; (b) $d=1 \mathrm{~mm}, P_{1}=4 \mathrm{hPa}$, (c) $d=1.5 \mathrm{~mm}$, $P_{1}=4 \mathrm{hPa},(\mathrm{d}) d=1 \mathrm{~mm}, P_{1}=0.8 \mathrm{hPa}$.

In Fig. 5 four images of ceramic grains on an adhesive tape taken with the use of the GSE detector at various gaseous working distances $d$ and gas pressures $P_{1}$ have been shown as an example. The first three images were taken at the relatively high pressure $4 \mathrm{hPa}$ of air used as the working gas. There, the gaseous working distances $d$ were changed gradually $(d=0.5,1$, and $1.5 \mathrm{~mm})$ which resulted in a gradual increase of the gas multiplication demonstrated as a rising brightness of sequential images. The fourth image was taken at the middle distance $d=1 \mathrm{~mm}$ and the relatively low working pressure. The pressure $0.8 \mathrm{hPa}$ was measured in the sample chamber but inside the space so close to the throttling aperture opening, the dynamical pressure must be much lower. Anyway, it was too low to produce enough ions for compensation of charging effects which are seen distinctly in Fig. 5d.

It should be also mentioned that an inseparable part of the combined detector system is an electronic multichannel amplifier unit. The unit must fulfill very rigor- ous requirements concerning its sensitivity, amplification, noise, frequency band, stability and many other features. In contrast to the semiconductor BSE detectors which effectively multiply highly energetic electrons, the GSE detector working at so minute $d$ distance provides low gas multiplication which must be supplemented by higher sensitivity of the amplifier channel.

\section{Conclusions}

The analyses of the GSED functioning at very low gaseous working distances were conducted to fit the detector parameters to the conditions of the combined $\mathrm{SE} / \mathrm{BSE}$ detector system. However, the problem of SE detection at the reduced gaseous working distance is even more essential for the investigations of wet biological samples which need much elevated pressure (even over $20 \mathrm{hPa}$ ) than that observed at the room temperature and should apply a possibly low voltage accelerating the electron beam (even below $5 \mathrm{kV}$ ) to obtain good contrast for the samples of low density. The presented results of computations and experiments are fully optimistic and prove the possibility of obtaining satisfying SE signals for the GSE detectors positioned as closely to the sample as the stability of the sample surface makes it possible. It should be also considered that the gaseous atmosphere in the observed region may be kept in the dynamic balance rather than in the stagnation state.

\section{Acknowledgments}

Electronic equipment for the detector used in this work has been designed by Mr. Jakub Brach. This work was supported with the budget resources for science in $2011 / 12$ as the research project.

\section{References}

[1] G.D. Danilatos, Adv. Electron. Electron Phys. 71, 109 (1988).

[2] G.D. Danilatos, Adv. Electron. Electron Phys. 78, 1 (1990).

[3] D.J. Stokes, Principles and Practice of Variable Pressure/Environmental Scanning Electron Microscopy (VP-ESEM), Wiley, Chichester 2008.

[4] W. Slówko, M. Krysztof, J. Microsc. 237, 292 (2010).

[5] M. Krysztof, W. Slówko, Acta Phys. Pol. A 120, 19 (2011). 